\title{
Obstetric outcomes with large fibroids in pregnancies nearing term: a study in a tertiary care centre in rural india
}

\begin{abstract}
Purpose: The exact incidence of large fibroids $(>5 \mathrm{~cm})$ complicating pregnancy is not known and the numbers are increasing. There are not enough studies with large fibroids in pregnancy as most of them find it difficult to conceive.

Methods: We present a prospective evaluation of a series of 15 patients with large fibroids $(>5 \mathrm{~cm})$ complicating pregnancies, who had delivered in our hospital over a period of 6 months. They had often not been booked in pregnancy earlier. We considered only those with large fibroids in the lower uterine segment and reviewed the related adverse maternal and fetal outcomes at or near term and any complications at post natal follow-up.

Results: All the women underwent caesarean deliveries. 8 of the $15(53 \%)$ women were at $34 \mathrm{yrs}$ of age and 13 out of the $15(87 \%)$ women had a history of infertility. 7 out of 15 women delivered prematurely (46\%). 2 women $(13.33 \%)$ with the largest fibroids measuring up to 16 and $24 \mathrm{~cm}$ respectively needed prophylactic bilateral internal iliac artery ligation and myomectomy to deliver the baby but avoided caesarean hysterectomies. Myomectomy could be avoided in the remaining 13 women. $60 \%$ women had fetal malpresentations. 9 out of 15 women $(60 \%)$ had postpartum hemorrhage and most of the women $(87 \%)$ needed blood transfusions. Posteriorly located cervical fibroids bled more than anterior cervical fibroids. Puerperal pyrexia was noted in $50 \%$ of the women with retained uterus.

Conclusion: Reduction of fibroid size pre-pregnancy could have avoided maternal and fetal complications which became inevitable once pregnancy reached advanced gestation. When women do present at term pregnancy with such large fibroids devascularisation techniques like prophylactic bilateral internal artery ligation may reduce blood loss and avoid caesarean hysterectomy.
\end{abstract}

Keywords: Fibroid; Leiomyoma; Caesarean myomectomy; Cesarean hysterectomy; Lower segment fibroid
Volume 7 Issue 2 - 2017

\author{
Anirban Dasgupta, Debjyoti Santra,Anirban \\ Talukdar, Utpal Ghosh, Sneha Narayan, \\ Subhendu Dasgupta \\ Department of Obstetrics \& Gynaecology (OBG), Bankura \\ Sammilani Medical College \& Hospital (BSMCH), India
}

Correspondence: Anirban Dasgupta, Department of Obstetrics \& Gynaecology (OBG), Bankura Sammilani Medical College \& Hospital (BSMCH), D-15, Sarojini Naidu Path, NonCompany, City Centre, Durgapur, West Bengal, India-713216, Tel 9|805646I564, Email dr.anirbandasgupta@gmail.com

Received: January 07, 2017 | Published: May 30, 2017

\section{Introduction}

The incidence of uterine leiomyomas in pregnancy is not uncommon, ranging from $1.6-10.7 \%$ depending on the size considered and the trimester of assessment. ${ }^{1}$ However the diagnosis is never straight forward with $42 \%$ of large fibroids $(>5 \mathrm{~cm})$ and $12.5 \%$ of small fibroids $(3-5 \mathrm{~cm})$ diagnosed by clinical examination. ${ }^{2}$ The increased thickness of the myometrium complicates the detection and hence the true prevalence of fibroids in pregnancy may be much higher. The overall incidence may be as high as $40-50 \%$ at age of $35 \mathrm{yrs}$ and 60 $70 \%$ at 40 yrs. $^{3}$

The exact incidence of large fibroids complicating pregnancy is not known and factors like increased success of IVF, as well as women opting for child birth at an advanced age after completing their careers influence such figures. There is a paucity of literature dealing with pregnancy complicated by large fibroids (particularly over lower uterine segment), as women with such large fibroids tend to have subfertility and any such pregnancy is fraught with chances of miscarriage. At an advanced gestation such high risk women also tend to have complications like anemia, antepartum hemorrhage (low lying placenta implanting over fibroid), fetal malpresentations, premature delivery, caesarean delivery(occasionally hysterectomy), postpartum hemorrhage, multiple blood transfusions among others. ${ }^{4}$ The obstetric care team have to anticipate great surgical challenges in such situations and these have also been dwelt on in this study.

\section{Materials \& methods}

We present a prospective evaluation of a total of 15 patients with fibroid complicating pregnancy at an advanced gestational age at or near term (Table 1). What made this study unique is that we included only those with large $(>5 \mathrm{~cm})$ fibroids involving at least the lower segment of the uterus. This cut-off figure of $5 \mathrm{~cm}$ was decided after a literature review. ${ }^{5}$ Noor S. ${ }^{6}$ suggested that the afore-mentioned adverse outcomes were significantly higher in this subgroup of fibroids complicating pregnancy.

The study was done over a period of 6 months from November 2014 till April 2015 in women who were admitted and delivered in Bankura Sammilani Medical College \& Hospital, a tertiary care hospital in a rural setting in eastern India. Approval was obtained from the institute ethics committee. Out of 8854 admissions and deliveries at or near term gestation in our institute during this period, we found 32 women with $>5 \mathrm{~cm}$ fibroids complicating pregnancy (prevalence: $0.36 \%$ ). We excluded 7 women in whom the fibroids were either subserosal/fundal and not involving the cervix/lower uterine segment; and a further 10 were excluded in whom the pregnancy resulted in miscarriage. We reviewed the history which was in unique in certain cases; the antenatal complications including anemia, antepartum hemorrhage, fetal malpresentations, prematurity, need for transfusions and the incidence of postpartum hemorrhage (PPH) as well as follow-up in postnatal period to rule out secondary PPH after retention of uterus. All 
the women underwent caesarean deliveries. Different modifications in the routine steps of caesarean section were necessary in the delivery of the babies due to altered anatomy of the lower segment of uterus, and these have been highlighted. The fair amount of limitations that we faced in this rural setting have also been discussed.

\section{Details of patients and challenges in delivery}

The patient specifics have been detailed in Table 1. There were 12 women with 5-10 cm fibroids in their lower uterine segment and 3 women with $>10 \mathrm{~cm}$ size fibroids. Those with smaller (around $5 \mathrm{~cm}$ lower segment fibroids) at term gestation with cephalic presentation and fundally located placenta were counselled regarding vaginal delivery but they opted for caesarean section, mostly because of previous history of infertility/bad obstetric history and the current pregnancy being a very precious conception. All the pregnancies were singleton and while 11 women had predominantly anterior wall lower segment fibroids, 4 of them had cervical fibroids extending laterally and posteriorly.

We would like to first highlight two patients ( $3 \& 10$ in Table 1$)$ with the largest (16-25cm) fibroids. Mrs. X (No. 3), 35yrs of age had conceived after $3 \mathrm{yrs}$ of primary infertility with b-thalassemia trait. She had a $24 \times 15 \mathrm{~cm}$ predominantly fundo-anterior intramural fibroid extending up to lower segment. After her combined screening revealed a risk of 1:100 for trisomy 21 , she opted for amniocentesis which revealed a normal karyotype. Her 20 weeks USG did not find any gross fetal anomalies but there was a low lying placenta implanted over the fibroid which persisted at subsequent USG at 28, 32 and 34 weeks. Serial fetal biometry revealed fetal growth appropriate for gestational age. She was admitted with vaginal bleeding from the placenta previa at 35 weeks. Following a course of corticosteroids we counselled her regarding high chances of caesarean delivery in view of major placenta previa and transverse lie of the baby. A high risk informed consent with arrangement of multiple blood products and chances of caesarean hysterectomy was explained and $\mathrm{Hb}$ levels optimised to $11 \mathrm{gm} \%$. Pre-operative planning regarding location of uterine incision was attempted based on USG findings. Under general anaesthesia, midline vertical sub-umbilical skin incision was given. In the absence of intervention radiology facilities, we opted to ligate the internal iliac arteies bilaterally to reduce blood loss. The huge fibroid was partially enucleated and brought out of abdominal cavity, before the myometrium below could be accessed for incision (Figure 1). The low lying placenta was avoided and did not need cutting through. The separation of the placenta and the removal of the fibroid from its bed resulted in around $800 \mathrm{ml}$ intra-operative bleedingfor which she received 1 unitblood transfusion intra-op and 1 unit post-op. A healthy baby of $2250 \mathrm{gms}$ was born. The prophylactic bilateral internal iliac artery ligation ensured hemorrhage was within manageable limits and a caesarean hysterectomy was avoided. She had an uneventful post op recovery with $\mathrm{Hb}$ of $10 \mathrm{gm} \%$ and the baby is doing well now. The histopathology of the fibroid (Figure 2) later showed areas of red degeneration. The other lady Mrs. Y (No.10) too had a pregnancy complicated with a large fibroid of $16 \mathrm{~cm}$ along with multiple smaller fibroids (Figure 3), breech presentation of the fetus, placenta previa and antepartum hemorrhage (APH) at 32 weeks. She underwent a caesarean section after bilateral internal iliac artery ligation following a course of antenatal corticosteroids. She received only 1 intraoperative blood transfusion.

The management of patients with fibroid size $5-10 \mathrm{~cm}$ was relatively straight forward and the uterine incisions to deliver the baby could be tailored according to their location in the pre-op USG. In some patients as in 3,5,9 and 11 (Table 1) even though the pre-op USG suggested a fibroid in the lower uterine segment (LUS), we could find space lower down in the LUS below the fibroid (Figure 4 area denoted by surgeon's finger) where the uterine incision was given to deliver the baby. This has also been demonstrated in other studies and case reports. ${ }^{7}$ Examination under anaesthesia helped to locate this space in the LUS by pushing the fibroid upwards (Figures $5 \& 6$ ). In others as in 1 and 12 , the baby was delivered by a classical caesarean section followed by repair of uterus in 3 layers with polyglactin 910 mattress. Importantly myomectomy at the time of caesarean section could be avoided for all these patients and further blood loss avoided.

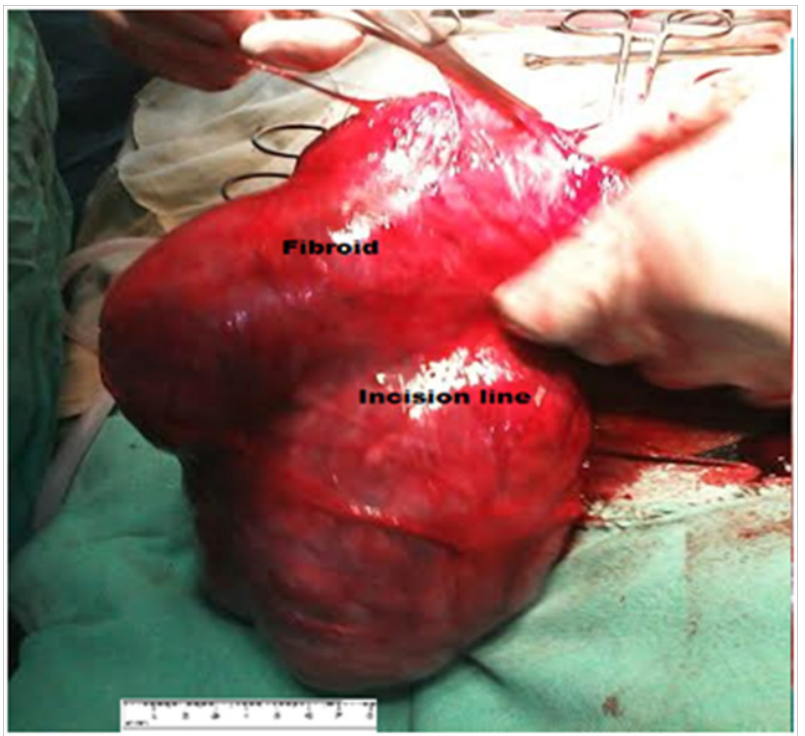

Figure I Uterine incision after fibroid enucleation.

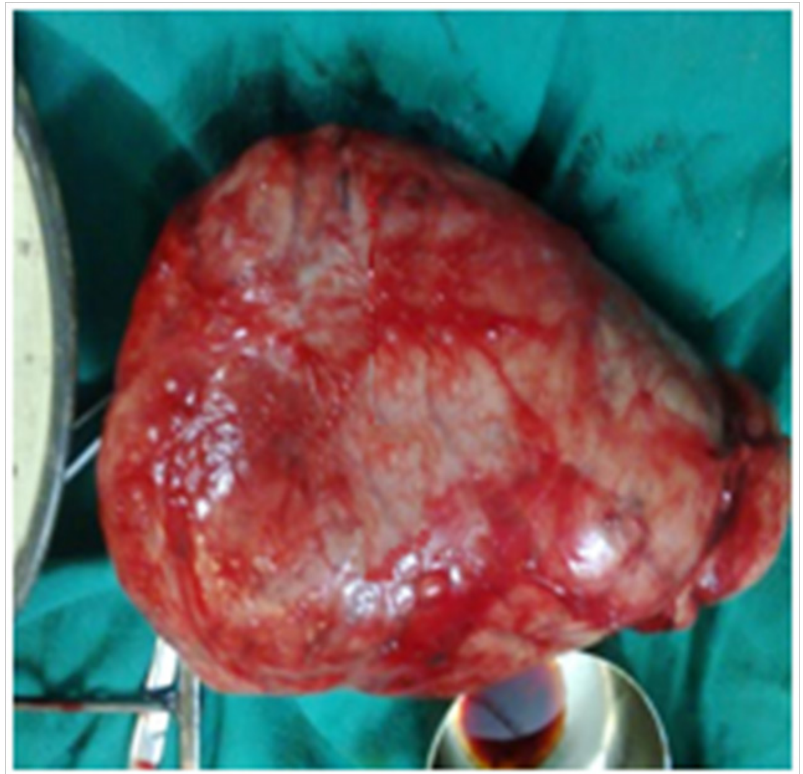

Figure 2 The fibroid after myomectomy (placental membrane being separated over the fibroid surface with the uterine incision below and medial to fibroid).

\section{Results}

8 of the $14(57 \%)$ women were $\geq 34$ yrs of age and 12 out of $15(80 \%)$ had a lower socio-economic status (Table 1). 13 out of the $15(87 \%)$ women had a history of infertility while two women with partly submucosal leiomyomas (4 \& 13 in Table 1$)$ had a history of 
2-3 late first trimester and early second trimester miscarriages (13\%) As mentioned earlier, all the pregnancies were singleton and while 11 women had predominantly anterior wall lower segment fibroids, 4 of them had cervical fibroids extending laterally and posteriorly.

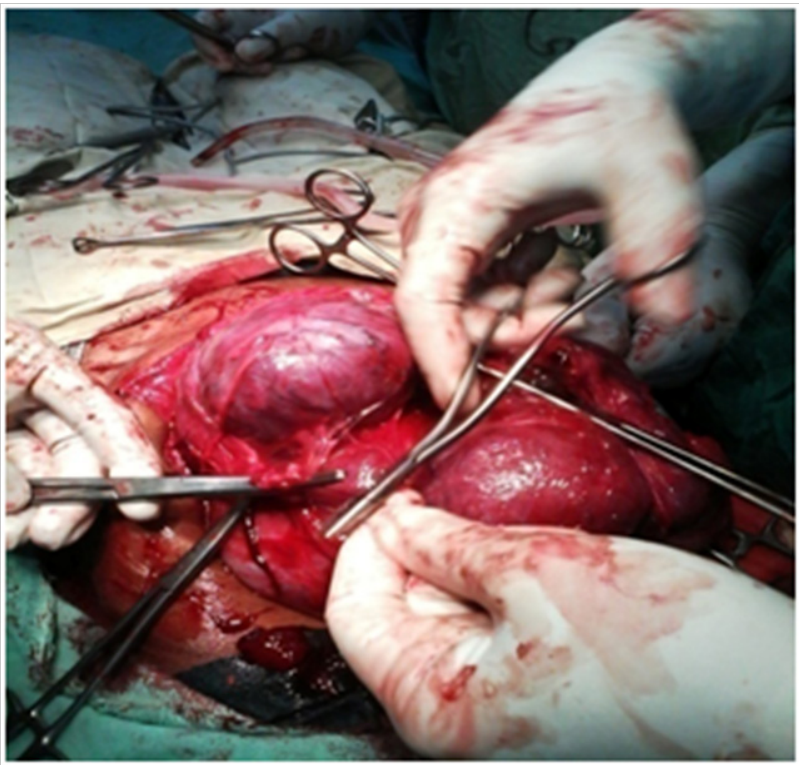

Figure 3 Multiple fibroids.

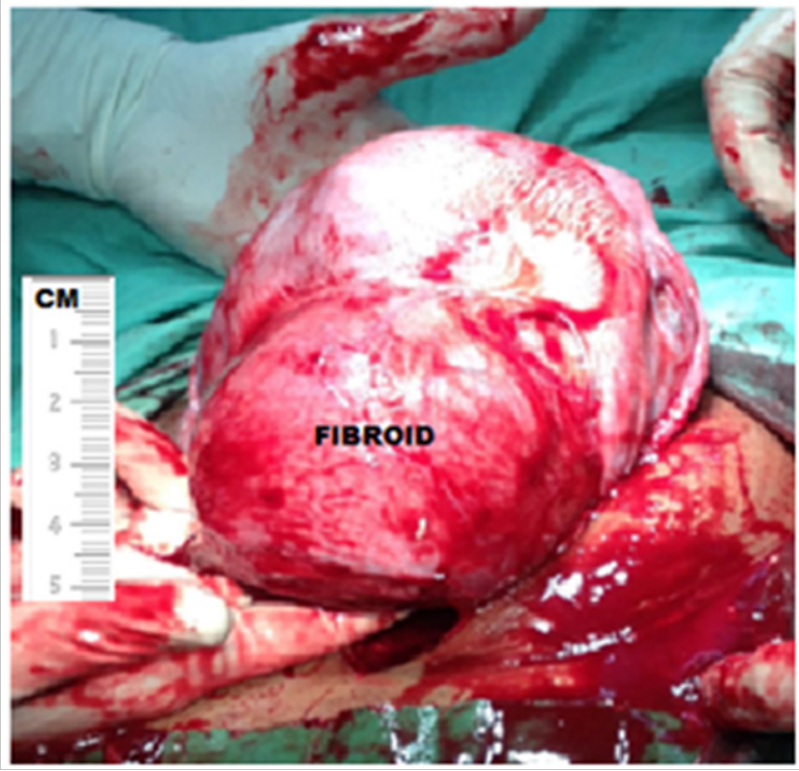

Figure 4 Uterine incision in LUS below fibroid.

7 out of 15 women delivered prematurely $(46 \%)$ and these included the 2 women with the largest fibroids and placenta previa. The mean gestational age of delivery was 35.33 weeks. Among the remaining women who delivered prematurely, no.1 underwent emergency classical c-section due to transverse lie with preterm premature membrane rupture (PPROM) at 34 weeks as did no. 13 but she also needed bilateral internal iliac artery ligation to control bleeding after separation of low lying placenta. The lady denoted as no.7 was an elderly primigravida at 35 weeks with a left anterolateral lower segment fibroid who was admitted in preterm labour with very poor antenatal care. The labour was prolonged and while an emergency c-section was done due to arrest in descent of fetal head, she developed PPH and received 2 units blood transfusion post- operatively. Numbers 9 and 14 received a course of corticosteroids and underwent preterm emergency c-sections at 33 and 31 weeks respectively after they presented with fetuses in breech presentation and PPROM and risked the chance of cord prolapse.

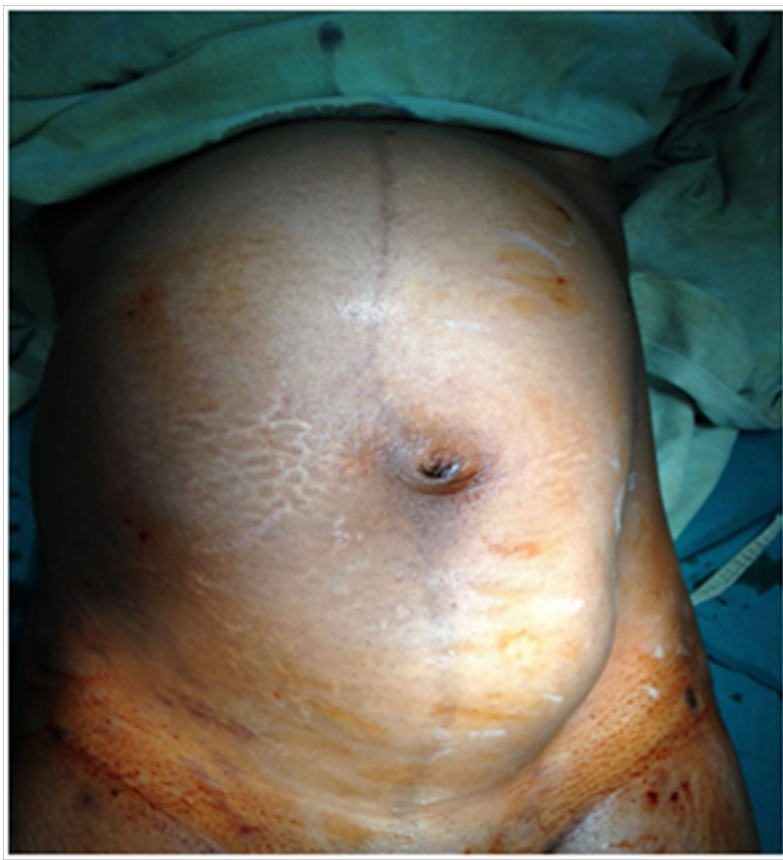

Figure 5 Arrow denoting location of lower segment fibroid in gravid uterus.

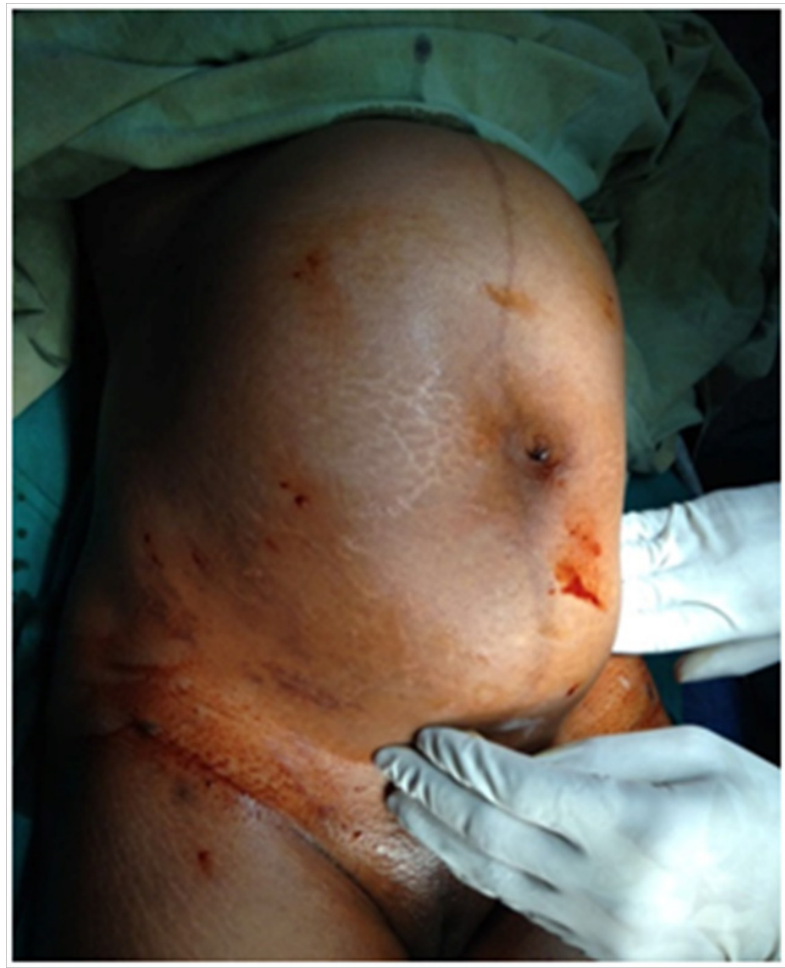

Figure 6 Palpation under anaesthesia to delineate space below fibroid in LUS

We also noticed that posteriorly located cervical fibroids (numbers $4,8 \& 13$ in Table 1) bled more (mean blood loss $850 \mathrm{ml}$ vs $510 \mathrm{ml}$ ) and also did not undergo much reduction in size in postnatal follow-up at 6-8 months compared to anterior cervical fibroids, the exact reason for which was not clear. 9 out of 15 women $(60 \%)$ had postpartum 
hemorrhage while 13 of the 15 women (87\%) received intra/post-op transfusions. 9 out of the 15 women had fetal malpresentations (60\%) among which 7 fetuses were in breech and 2 were in transverse lie. There was a significant reduction in size of the fibroids in 6 out of 12 $(50 \%)$ of the women with retained uterus in follow-up till 12 months. Inspite of earnest counselling, 2 of the women (2 and 6) conceived again after 1 year.

In our study we did not come across any gross fetal complications other than prematurity which was seen in 13 of the 15 babies. All babies were live born and in spite of the prenatal corticosteroids, we noticed Hyaline Membrane Disease in 2 of the babies (no.10 and14) and transient tachypnoea of newborn noticed in 8 of them. There was difficulty in delivering 2 of the babies with malpresentations particularly with transverse lie but they did not suffer any bone fracture/soft tissue trauma. Care was taken to grasp their legs first, that facilitated delivery with breech/transverse/oblique lie. All the babies were doing well postnatally at 6 months except one who had pneumonia at 3 months of age and did not survive.

In some patients we came across rare complications like a postop subphrenic collection in case 1 in whom we performed an USG guided drainage and she recovered uneventfully in the post-op period. In some others where uterus could be retained, we noticed a heavier lochia post-op but only in patient 8 did it manifest with secondary post partum hemorrhage, that was controlled with large bore intrauterine Foley catheter balloon inflation with $60-80 \mathrm{ml}$ normal saline. Puerperal pyrexia was noticed in 6 out of the 12 women (50\%) with retained uterus which were managed conservatively.

\section{Discussion}

Inspite of the increased incidence of fibroids in pregnancy, the relationship of fibroids and adverse outcomes in pregnancy are not clearly understood. Around a third do increase in size with $60-78 \%$ showing no change in volume during pregnancy. ${ }^{2}$ For those that grew in size, the growth was mostly seen in the first trimester. Mostly the fibroids are asymptomatic with only $9 \%$ of large fibroids showing any symptoms; mainly lower abdominal pain in the 2 nd or 3 rd trimesters suggestive of red degeneration. Vice versa around 10-30\% fibroids have complications in pregnancy. ${ }^{3}$ Early miscarriages are more common with multiple fibroids and more often than not when they are located in the body of the uterus than in the lower uterine segment. ${ }^{8}$ and in intramural and submucosal fibroids. ${ }^{9}$. Bleeding in first trimester is seen if the placenta implants close to the fibroid, and the incidence of preterm labour, if not preterm premature rupture of membranes, is also increased. Ciavattini et al.${ }^{10}$ demonstrated that the risk of preterm labour is significantly increased with both multiple fibroids $(29.4 \%$ vs $5 \%$ in women with no fibroids) as well as with large fibroids $>5 \mathrm{~cm}(16.4 \%$ vs $5 \%) .{ }^{10}$ Shavell. ${ }^{5}$ also demonstrated the earlier mean gestational age of delivery in pregnancies complicated by $>5 \mathrm{~cm}$ fibroids against subgroups of smaller fibroids $(1-5 \mathrm{~cm})$ and no fibroids ( 36.5 vs 38.4 vs 36.5 weeks). ${ }^{5}$

A submucosal, retroplacental fibroid may also contribute to placental abruption while the incidence of placenta previa is also increased 2 fold. ${ }^{9}$ Large, multiple fibroids as well as lower segment fibroids increase chances of malpresentations (OR:2.9) and hence caesarean sections (OR:3.7)(10). Multiple fibroids in pregnancy increase caesarean section $(73.5 \%$ vs $37 \%, \mathrm{p}<0.01)$ and breech presentation rates $(11.8 \%$ vs $2.7 \%, \mathrm{p}=0.04) .{ }^{10}$ Blood loss at delivery is increased more so for larger $>5 \mathrm{~cm}$ fibroids than smaller fibroids and normal pregnancy $(645 \mathrm{ml}$ vs $535 \mathrm{ml}$ vs $486 \mathrm{ml}) .{ }^{5}$ Uterine atony was also more significant with fibroids than without (12.3 vs $4.2 \%) .{ }^{11}$ One has to guard against post partum hemorrhage in such a scenario and chances of a caesarean hysterectomy.

Myomectomy at the time of caesarean delivery causes severe hemorrhage and should be reserved only for subserous, pedunculated fibroids and in select cases of submucous fibroids. ${ }^{12}$ However certain authors (Machado and colleagues) have demonstrated relative safety of caesarean myomectomy with fibroids $>5 \mathrm{~cm}$ size in experienced hands in tertiary care centres with step wise devascularisation and use of intra-uterine Foley's balloon catheter use. ${ }^{13}$ Sultana et al.. ${ }^{14}$ in a series of 98 women also demonstrated no additional blood loss or increase in length of hospital stay with caesarean myomectomy but found doubling of operating time without requirement of caesarean hysterectomy. However the largest fibroid size was limited to $12 \mathrm{~cm}$ in this study. Yet another smaller retrospective study with 16 women arrived at similar conclusions. ${ }^{15}$

Pregnancy as such leads to gradual reduction in the size of fibroids, a process that may continue post-partum. Laughlin et al. ${ }^{16}$ in fact showed in his study of 171 women with a single initial fibroid, that $36 \%$ did not show any identifiable fibroid at the time of postpartum USG while $79 \%$ of the remaining underwent considerable reduction in size. There have also been rare isolated case reports of large lower segment fibroid being expelled as a polypoidal mass post partum after caesarean section as demonstrated by Barsode et al. ${ }^{17}$

\section{Conclusion}

Women undergoing evaluation for infertility often undergo treatment for large fibroids mostly. However in our rural setting due to lack of proper imaging studies/intervention radiology, surgical measures like prophylactic bilateral internal iliac artery ligation helped in reducing surgical and post-operative morbidity. Consultant led care, an honest session of counselling for the patient and her relatives and explaining the increased chances of caesarean hysterectomy, early arrangement of blood products and other eventualities need to be discussed. It is imperative that the lead consultant be involved in all such caesarean deliveries with the lead anaesthetist along with the paediatrician to facilitate transfer of the baby to SNCU in case of suspected prematurity. A level 2 ICU bed is kept ready should the need arise. The lady and her relatives are debriefed in the postnatal follow-up with emphasis to return immediately if she notices heavy vaginal bleeding in the puerperium. Contraceptive advice along with the need of adequate spacing of child birth need to be explained. These women also are told about the increased risk of placenta previa and accreta with subsequent caesarean sections in future pregnancies.

\section{Acknowledgments}

None.

\section{Conflicts of interest}

None.

\section{References}

1. Pregnancy in women with uterine leiomyomas (fibroids).

2. Lee HJ, Norwitz ER, Shaw J. Contemporary Management of Fibroids in Pregnancy. Rev Obstet Gynecol. 2010;3(1):20-27.

3. Katz VL, Dotters DJ, Droegemeuller W. Complications of uterine leiomyomas in pregnancy. Obstet Gynecol. 1989;73(4):593-596.

4. Parker WH, Olive DL, Pritts EA. Fibroids and pregnancy outcomes. Fertil Steril. 2012;98(1):e13. 
5. Shavell VI, Thakur M, Sawant A, et al. Adverse obstetric outcomes associated with sonographically identified large uterine fibroids. Fertil Steril. 2012;97(1):107-110.

6. Noor S, Fawwad A, Sultana R, et al. Pregnancy with fibroids and its and its obstetric complication. J Ayub Med Coll Abbottabad. 2009;21(4):3740 .

7. C Jothikala, Jamila Hameed, S Radhika, et al. Lower Uterine Segmen Fibroid Complicating Pregnancy: A Case Report. JSS Case Reports \& Reviews. 2014;1(6):18-19.

8. Lev-Toaff AS, Coleman BG, Arger PH, et al. Leiomyomas in pregnancy: sonographic study. Radiology. 2014;164(2):375-380.

9. Klatsky PC, Tran ND, Caughey AB, et al. Fibroids and reproductive outcomes: a systematic literature review from conception to delivery. Am J Obstet Gynecol. 2008;198(4):357-366.

10. Ciavattini A, Clemente N, Delli Carpini G, et al. Number and size of uterine fibroids and obstetric outcomes. J Matern-Fetal Neonatal Med. 201528(4):484-488.
11. Morgan Ortiz F, Piña Romero B, Elorriaga García E, et al. [Uterine leiomyomas during pregnancy and its impact on obstetric outcome]. Ginecol Obstet Méx. 2011;79(8):467-473.

12. Ouyang DW, Economy KE, Norwitz ER. Obstetric complications of fibroids. Obstet Gynecol Clin North Am. 2006;33(1):153-169.

13. Machado LS, Gowri V, Al-Riyami N, et al. Caesarean Myomectomy: Feasibility and safety. Sultan Qaboos Univ Med J. 2012;12(2):190-196.

14. Sultana R, Noor S, Nazar AF, et al. Safety of caesarean myomectomy. $J$ Ayub Med Coll Abbottabadv. 2012;24(2):120-121.

15. Incebiyik A, Hilali NG, Camuzcuoglu A, et al. Myomectomy during caesarean: a retrospective evaluation of 16 cases. Arch Gynecol Obstet. 2014;289(3):569-573.

16. Laughlin SK, Herring AH, Savitz DA, et al. Pregnancy-related fibroid reduction. Fertil Steril. 2010;94(6):2421-2423.

17. Salil Barsode, Vaishali Taralekar, Tushar Panchanadikar. A Rare Case of Massive Lower Uterine Segment Fibroid with Pregnancy with Spontaneous Postpartum Expulsion. Journal of South Asian Federation of Obstetrics and Gynaecology. 2013;5(3):166-167. 